\title{
Performance on the American Board of Family Medicine Certification Examination by Country of Medical Training
}

\author{
John L. Falcone, and Donald B. Middleton
}

Background: Performance on the American Board of Family Medicine (ABFM) Certification and Recertification Examinations by country of medical school training has not been examined. Based on internal medicine patterns, we hypothesize that examinees trained in the United States and Canada would outperform examinees trained in other countries.

Methods: In this retrospective cohort study from 2004 to 2011, data on the ABFM examinations were obtained from the ABFM. Fisher exact and $\chi^{2}$ tests were performed across years based on the country of examinee training. Simple linear regression was performed to evaluate pass rates over time. All statistics were performed using an $\alpha=0.05$.

Results: The overall pass rate over the study period was $84.4 \%(74,821$ of 88,680$)$. The pass rate for US medical graduates (USMGs) was $88.3 \%(60,328$ of 68,332$)$. The pass rate for Canadian medical graduates (CMGs) was $93.8 \%$ (872 of 930). The pass rate for non-Canadian foreign medical gradates (NC-FMGs) was 70.1\% $(13,621$ of 19,418). CMGs had a higher pass rate than USMGs $(P<.001)$ and NC-FMGs $(P<.001)$. Simple linear regression showed significant decreasing trends over time for all examinees $(P=.02)$, for USMGs $(P=.02)$, and for CMGs $(P=.02)$.

Conclusions: USMGs and CMGs outperform NC-FMGs on the ABFM certification and recertification examinations. These findings may alter acceptance patterns for Family Medicine residency programs. (J Am Board Fam Med 2013;26:78-81.)

Keywords: Certification, Educational Measurement, Geographic Factors, Medical Education

Passing the American Board of Family Medicine (ABFM) Certification Examination (CE) is required for both initial board certification and board recertification. The CE is a computer-based, multiple-choice examination that tests medical knowledge and problem-solving ability relevant to the practice of Family Medicine. The passing level is not based on failing or passing a particular percent-

This article was externally peer reviewed.

Submitted 15 August 2012; revised 12 October 2012; accepted 18 October 2012.

From the University of Pittsburgh School of Medicine, University of Pittsburgh Medical Center, Department of Surgery (JLF); and University of Pittsburgh School of Medicine, University of Pittsburgh Medical Center, St. Margaret Hospital, Department of Family Medicine (DBM), Pittsburgh, PA.

Funding: none.

Conflict of interest: none declared.

Corresponding author: John L. Falcone, University of Pittsburgh Medical Center, Presbyterian University Hospital, F-675, 200 Lothrop Street, Pittsburgh, PA 15213 (E-mail falconej@upmc.edu). age of candidates, but rather on a score that demonstrates a consensus level of mastery of the vast field of Family Medicine. Certification and recertification candidates take the same examination. ${ }^{1}$

The published literature about demographic predictors of performance on the ABFM CE is sparse. One regional study from the 1980s demonstrated no difference in performance between university-based and community-based Family Medicine residency programs. ${ }^{2}$ Performance on the ABFM CE by country of medical school training has not been examined. In a related specialty, performance on the American Board of Internal Medicine (ABIM) CE has been evaluated based on the country of medical training. ${ }^{3-7}$ US medical graduates (USMGs) had significantly higher pass rates compared with foreign medical graduates (FMGs). As a corollary, US citizens who graduated from US programs had higher pass rates compared with US citizens who graduated from a foreign medical 
school. ${ }^{4-6}$ In addition, a higher percentage of US and Canadian medical school graduates in a residency class is associated with better performance for that residency program on the ABIM CE. ${ }^{7}$ The performance of certified internists on the ABIM CE has been positively correlated with clinical skills. ${ }^{8}$

With the superior performance of examinees trained in Internal Medicine at medical schools in the United States and Canada, we hypothesize that certification and recertification candidates medically trained in the United States and Canada will have higher performance on the ABFM CE than certification and recertification candidates medically trained in other countries.

\section{Methods}

In this retrospective cohort study from 2004 to 2011, the publicly available electronic data for performance on the ABFM CE for initial certification and recertification were obtained from the $\mathrm{ABFM}$ website. ${ }^{9}$ The outcomes of the ABFM CE by country of medical training over the study period were evaluated for the following groups: USMGs, FMGs, Canadian medical graduates (CMGs), and non-Canadian FMGs (NC-FMGs). Academic years were included if complete data were available for review. Permission to cite and use published electronic data for research purposes was confirmed with the Vice President of Psychometric Services for the ABFM.

Longitudinal performance for groups was shown by graphing pass rates over time. One-tailed $\chi^{2}$ tests and Fisher exact tests were used to test for differences between groups. Comparisons were performed for each individual year and over the entire study period. To show trends in annual pass rates, simple linear regression was performed with the year as the independent variable and the pass rate as the dependent variable. All statistics were performed using Stata 11.1 statistical software (StataCorp, College Station, TX), using an $\alpha=0.05$.

\section{Results}

Certification and recertification examination outcomes by country were available from 2004 to 2012. The year 2012 had incomplete data and did not satisfy inclusion criteria for this study. A total of 88,680 unique examination results were evaluated from 2004 to 2011. The overall pass rate for all examinees over the study period was $84.4 \%(74,821$ of 88,680$)$. The pass rate for USMGs was $88.3 \%$
(60,328 of 68,332), for CMGs was 93.8\% (872 of 930), and for NC-FMGs was $70.1 \%(13,621$ of 19,418). $\chi^{2}$ Tests between passing and failing examinees showed that CMGs had a higher overall pass rate than USMGs $(P<.001)$ and NC-FMGs $(P<.001)$. A $\chi^{2}$ test between passing and failing examinees showed USMGs had a higher overall pass rate than FMGs $(P<.001)$ and NC-FMGs $(P<.001)$.

Longitudinal pass rates are shown in Figure 1. $\chi^{2}$ Analyses showed that USMGs had a higher pass rate than FMGs and NC-FMGs for each year of the study period (all $P<.001$ ) and overall (both $P<.001)$. These analyses also showed that CMGs had a higher pass rate than USMGs in $2004(P<$ $.001), 2006(P=.02), 2009(P=.02)$, and overall $(P<$ $.001)$. There were no differences in 2005,2007 to 2008, and 2010 to 2011 (all $P>.05$ ). Finally, the $\chi^{2}$ analyses showed that $\mathrm{CMG}$ had a higher pass rate than NC-FMGs from 2004 to $2009(P<.001)$, in $2011(P=.001)$, and overall $(P<.001)$. There were no differences in $2010(P=.07)$.

Simple linear regression using the year as the independent variable and the pass rate as the dependent variable showed significant decreasing examination trends over time for all examinees $(P=.02)$, for USMGs $(P=.02)$, and for CMGs $(P=.02)$. There were no significant examination trends over time for FMGs $(P=.21)$ or NC-FMGs $(P=.26)$.

\section{Discussion}

In this study, we have demonstrated that USMGs and CMGs have higher rates of passing the ABFM CE for initial board certification and recertification than NC-FMGs. This finding is consistent with our study hypothesis and with conclusions in the published Internal Medicine literature. ${ }^{3-8}$ CMGs have higher overall pass rates than USMGs. This difference is possibly because of the lower numbers of CMGs compared with USMGs. Statistically significant differences occur in the overall comparison but not for each year of the study period. The reasons behind the differences in examinee performance are ultimately unknown. There are likely differences in the international medical school curricula, and these differences should be studied in the future.

We also found statistically significant decreasing pass rate trends for USMGs and CMGs. This finding is very concerning. A similar decreasing pass rate trend has been seen recently on the American Board 
Figure 1. Longitudinal pass rates.

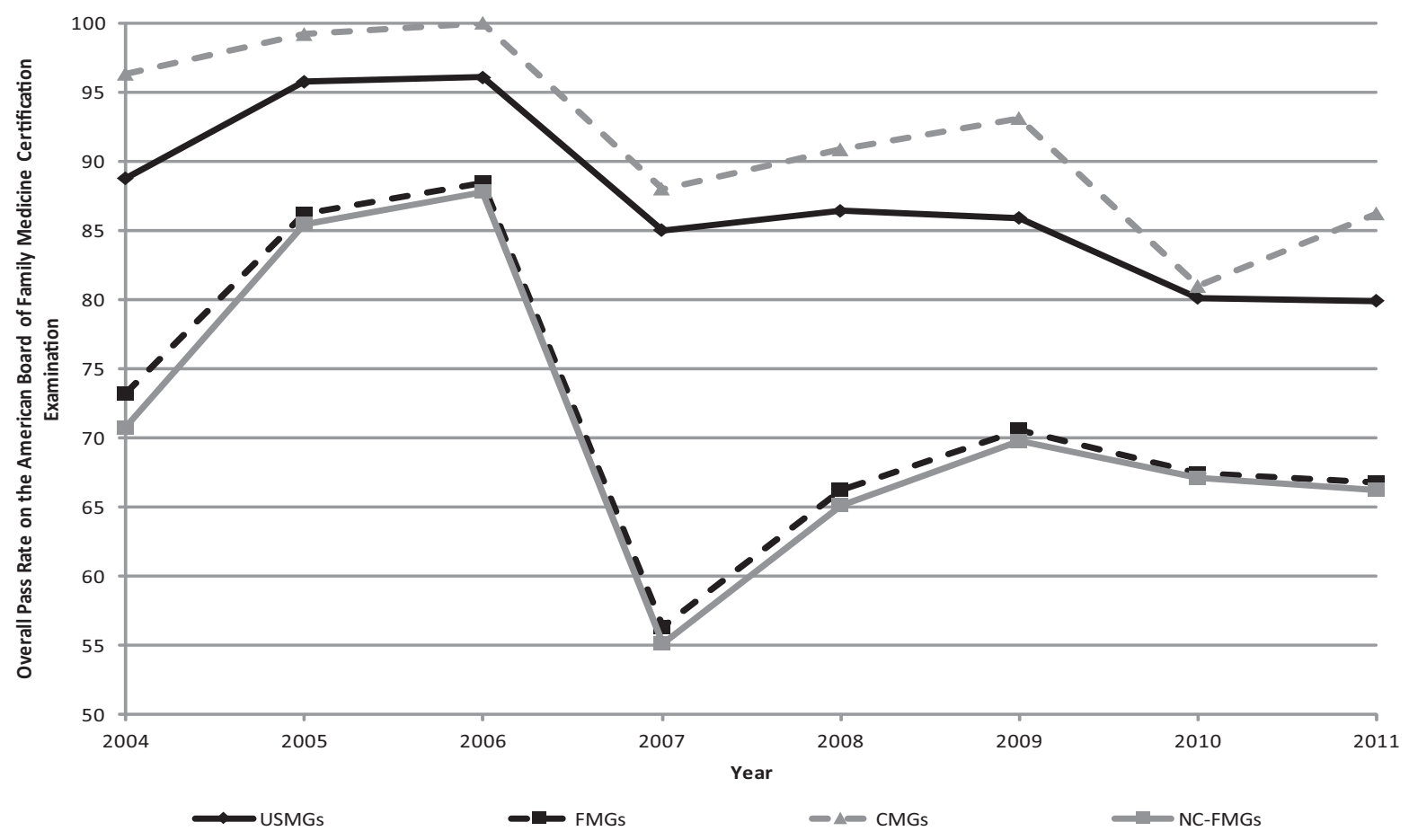

CMGs: Canadian Medical Graduates

FMGs: Foreigh Medical Graduates

NC-FMGs: Non-Canadian Foreign Medical Graduates

USMGs: United States Medical Graduates

of Surgery Certifying Examination. ${ }^{10}$ The explanation for these decreasing trends on the ABFM CE is unclear. The examination blueprint changed in 2006 from the classic test theory psychometric model to one that relies on item response theory. This significant change was felt to be necessary to direct relevant field content while focusing on the knowledge required to practice a higher quality of family medicine. ${ }^{11}$ However, this chang in examination blueprint did not go into effect until a few years into the study period. This study would suggest that the decreasing pass rate trend had been significant even before the blueprint changed. Further examination of these trends and efforts to determine the root cause is warranted and recommended.

There are several notable limitations to this study. First, because our study is retrospective, a cause and effect for the results is not able to be determined. Confounding variables such as the applicant quality, medical school and residency program curricula, and ability to attract effective teaching staff or clinical material are likely some of the reasons behind our findings. Second, initial certification candidates could not be differentiated from recertification candidates. Even though both groups of candidates took the same examination, there are inherent differences between these groups. Third, it is unclear where examinees completed residency training. This may have an impact on examination outcome. Finally, there were no data available for the age or length of time since medical school and residency training for recertification examinees.

These limitations are a source of future directions with regard to research related to the ABFM CE. Separating initial certification candidates from recertification candidates would make for more specific and homogenous study groups. Further research looking at residency training would be worthwhile to describe other potential demographic predictors of ABFM CE performance for initial certification and recertification. In addition, evaluating time from medical school and residency training would give insight into how timing factors are important for recertification candidates.

Strengths of this study include its relatively straightforward design. Because the examination re- 
sults used here are available publically, this study could easily be repeated. Our study is the first to show that the location of medical school training can affect board examination outcomes in Family Medicine for initial certification and recertification. This study also incorporates the entire population of examinees as opposed to a cohort of local or regional examinees.

\section{Conclusion}

Overall, we found that for board certification and recertification, USMGs and CMGs outperform NC-FMGs on the ABFM CE. We also found significant decreasing pass rate trends over time for multiple cohorts of examinees. Because pass rates on the ABFM CE are important, the results of this study have real potential to impact the interview and acceptance patterns of applicants to Family Medicine residency programs.

\section{References}

1. American Board of Family Medicine. Examination descriptions. Available from: https://www.theabfm. org/moc/exams.aspx. Accessed 19 June 2012.

2. Davidson RC, Kahn NB Jr. A comparison of university-based and community-based family practice residency programs. J Fam Pract 1984;18:581-6.

3. Meskauskas JA, Benson JA Jr, Hopkins E. Performance of graduates of foreign medical schools on the examinations of the American Board of Internal Medicine. N Engl J Med 1977;297:808-10.

4. Norcini JJ, Kimball HR, Grosso LJ, Day SC, Baranowski RA, Horne MW. Certification in internal medicine: 1989-1992. J Gen Intern Med 1994;9:361-5.

5. Norcini JJ, Shea JA, Webster GD, Benson JA Jr. Predictors of the performance of foreign medical graduates on the 1982 certifying examination in internal medicine. JAMA 1986;256:3367-70.

6. Benson JA Jr, Meskauskas JA, Grosso LJ. Performance of U.S. citizen-foreign medical graduates on certifying examinations in internal medicine. Am J Med 1981;71:270-3.

7. Norcini JJ, Grosso LJ, Shea JA, Webster GD. The relationship between features of residency training and ABIM certifying examination performance. J Gen Intern Med 1987;2:330-6.

8. Ramsey PG, Carline JD, Inui TS, Larson EB, LoGerfo JP, Wenrich MD. Predictive validity of certification by the American Board of Internal Medicine. Ann Intern Med 1989;110:719-26.

9. American Board of Family Medicine. Examination pass rates. Available from: https://www.theabfm.org/ moc/passrate.aspx. Accessed 19 June 2012.

10. Falcone JL, Hamad GG. The American Board of Surgery Certifying Examination: a retrospective study of the decreasing pass rates and performance for first-time examinees. J Surg Educ 2012;69:231-5.

11. Norris TE, Rovinelli RJ, Puffer JC, Rinaldo J, Price DW. From specialty-based to practice-based: a new blueprint for the American Board of Family Medicine cognitive examination. J Am Board Fam Pract $2005 ; 18: 546-54$. 